\title{
Ranking of Trapezoidal Bipolar Fuzzy Numbers Based on a New Improved Score Function
}

\author{
Jeevaraj $\mathrm{S}^{\text {a, } 1}$ \\ a Atal Bihari Vajpayee Indian Institute of Information Technology and Management \\ Gwalior, Madhya Pradesh, India-474015
}

\begin{abstract}
Bipolar fuzzy numbers plays a vital role in any Decision-making problem modelled under a bipolar fuzzy environment. In 2018, Akram and Arshad [1] introduced a new ranking function on the class of Trapezoidal Bipolar fuzzy numbers based on the area of the left and right membership function of a TrBFN, and they have discriminated any two TrBFNs by using it. The ranking principle introduced by Akram and Arshad [1] works better only when two bipolar fuzzy numbers have different rankings. We describe that the ranking function does not work with counterexamples when two or more bipolar fuzzy numbers have the same rankings. In this paper, we improve the ranking principle introduced in [1] by introducing a new Improved Score function. Firstly, we discuss the drawbacks and limitations of the ranking function introduced by Akram and Arshad [1]. Secondly, we introduce a new ranking function and study its properties. Thirdly, we introduce a new ranking principle by combining Akram and Arshad's [1] ranking function and the proposed ranking function. Finally, we show the efficiency of the proposed ranking principle in comparing arbitrary TrBFNs.
\end{abstract}

Keywords. Trapezoidal Bipolar fuzzy number, Ranking, Triangular Bipolar fuzzy number, Improved score function

\section{Introduction}

Fuzzy numbers play a major in solving problems involving imprecise numerical quantity. Further, it has been generalized to various forms such as intuitionistic fuzzy numbers, Pythagorean fuzzy numbers, Fermatean fuzzy numbers, Bipolar fuzzy numbers, etc. Various ranking procedures are available on the different classes of fuzzy and intuitionistic fuzzy numbers $[1,2,3,5,6,7,8,9,10,11]$. Bipolar fuzzy numbers are very much valuable for modelling problems with imprecise and incomplete information. Especially, Trapezoidal (Triangular) Bipolar Fuzzy numbers (TrBFN) are widely used in the literature $[1,4,12,13]$ for uncertainty modelling, and sometimes it performs better than the trapezoidal fuzzy numbers and trapezoidal intuitionistic fuzzy numbers. Ranking of Trapezoidal Bipolar fuzzy numbers plays a vital role in any Decision-making problem mod-

\footnotetext{
${ }^{1}$ Corresponding Author: Assistant Professor in Mathematics, Atal Bihari Vajpayee Indian Institute of Information Technology and Management Gwalior, Madhya Pradesh, India-474015; Email:jeevaraj@iiitm.ac.in
} 
elled under a bipolar fuzzy environment. Reza et al. [11] discussed the idea of duality in Linear programming problems. Rayappan and Mohana discuss the applications of spherical fuzzy sets in solving the MCDM problem. Akram and Arshad [1] has derived a new ranking principle for ordering any two Trapezoidal (Triangular) Bipolar Fuzzy Numbers (TrBFN), and they have compared any two Trapezoidal (Triangular) Bipolar Fuzzy Numbers by using the same ranking principle. They have also developed a group decision-making method based on trapezoidal bipolar fuzzy TOPSIS. Using the formula (3.3) and formula (3.4) on page number 574 (of Akram and Arshad [1]), they have shown that they can compare any two Trapezoidal (Triangular) Bipolar Fuzzy Numbers utilizing proposed ranking function $R_{f 1}, R_{f 2}$. However, their method does not compare any two arbitrary TrBFNs effectively. Highly motivated by the work of Akram and Arshad [1], firstly, we introduce a new improved score function on the class of TrBFNs and study its properties. Then, we present a new ranking principle by combining Akram and Arshad's [1] ranking function and the proposed ranking function. Finally, we show the efficiency of the proposed ranking principle in comparing arbitrary TrBFNs.

\section{Preliminaries}

Here we give some of the basic definitions defined on the class of TrBFNs.

Definition 2.1. Akram and Arshad [1].

A Bipolar Fuzzy Number (BFN) $A=\langle I, K\rangle=\left\langle\left[t_{1}, t_{2}, t_{3}, t_{4}\right],\left[k_{1}, k_{2}, k_{3}, k_{4}\right]\right\rangle$ is a Trapezoidal Bipolar Fuzzy Number (TrBFN), denoted by $\left\langle\left(t_{1}, t_{2}, t_{3}, t_{4}\right),\left(k_{1}, k_{2}, k_{3}, k_{4}\right)\right\rangle$, if its satisfaction degree $\lambda_{I}$ and dissatisfaction degree $\lambda_{K}$ are given as:

$\lambda_{I}=\left\{\begin{array}{ll}\frac{x-t_{1}}{t_{2}-t_{1}} & \text { if } x \in\left[t_{1}, t_{2}\right] \\ 1 & \text { if } x \in\left[t_{2}, t_{3}\right] \\ \frac{t_{4}-x}{t_{4}-t_{3}} & \text { if } x \in\left[t_{3}, t 4\right] \\ 0 & \text { Otherwise }\end{array}\right.$ and $\lambda_{K}= \begin{cases}\frac{k_{1}-x}{k_{2}-k_{1}} & \text { if } x \in\left[k_{1}, k_{2}\right] \\ -1 & \text { if } x \in\left[t_{2}, t_{3}\right] \\ \frac{x-k_{4}}{k_{4}-k_{3}} & \text { if } x \in\left[k_{3}, k 4\right] \\ 0 & \text { Otherwise }\end{cases}$

Note: Here, $t_{1} \leq t_{2} \leq t_{3} \leq t_{4}$ and $k_{1} \leq k_{2} \leq k_{3} \leq k_{4}$.

Definition 2.2. Akram and Arshad [1].

A BFN, $A=\langle I, K\rangle=\left\langle\left[t_{1}, t_{2}, t_{3}, t_{4}\right],\left[k_{1}, k_{2}, k_{3}, k_{4}\right]\right\rangle$ is a Triangular Bipolar Fuzzy Number (TBFN), denoted by $\left\langle\left(t_{1}, t_{2}, t_{3}\right),\left(k_{1}, k_{2}, k_{3}\right)\right\rangle$, if its satisfaction degree $\lambda_{I}$ and dissatisfaction degree $\lambda_{K}$ are given as:

$\lambda_{I}=\left\{\begin{array}{ll}\frac{x-t_{1}}{t_{2}-t_{1}} & \text { if } x \in\left[t_{1}, t_{2}\right] \\ \frac{t_{3}-x}{t_{3}-t_{2}} & \text { if } x \in\left[t_{2}, t 3\right] \\ 0 & \text { Otherwise }\end{array}\right.$ and $\lambda_{K}= \begin{cases}\frac{k_{1}-x}{k_{2}-k_{1}} & \text { if } x \in\left[k_{1}, k_{2}\right] \\ \frac{x-k_{3}}{k_{3}-k_{2}} & \text { if } x \in\left[k_{2}, k 3\right] \\ 0 & \text { Otherwise }\end{cases}$

Note: Here, $t_{1} \leq t_{2} \leq t_{3}$ and $k_{1} \leq k_{2} \leq k_{3}$.

Definition 2.3. Akram and Arshad [1].

Ranking Function $R_{f 1}$ for $\operatorname{TrBFN}$

Let $A=\langle I, K\rangle=\left\langle\left(t_{1}, t_{2}, t_{3}, t_{4}\right),\left(k_{1}, k_{2}, k_{3}, k_{4}\right)\right\rangle$ be the TrBFN. Then by using Definition 2.1, they have derived the ranking function $R_{f 1}$ of $\operatorname{TrBFN}$ as $R_{f 1}(A)=$ $(m(I)+\sigma(I)-(m(K)+\sigma(K)))$, where the means $(m(I), m(K))$ are defined as $m(I)=$ $\frac{t_{1}+t_{2}+t_{3}+t_{4}}{4}, m(K)=\frac{k_{1}+k_{2}+k_{3}+k_{4}}{4}$ and the areas $(\sigma(I), \sigma(K))$ are defined as $\sigma(I)=$ 
$\frac{-t_{1}-t_{2}+t_{3}+t_{4}}{2}$ and $\sigma(K)=\frac{-k_{1}-k_{2}+k_{3}+k_{4}}{2}$.

Therefore, $R_{f 1}(A)=\frac{\left(-t_{1}-t_{2}+3 t_{3}+3 t_{4}\right)-\left(-k_{1}-k_{2}+3 k_{3}+3 k_{4}\right)}{4}=\frac{-t_{1}-t_{2}+3 t_{3}+3 t_{4}+k_{1}+k_{2}-3 k_{3}-3 k_{4}}{4}$. If $\sigma(I), \sigma(K) \geq 1$, then the ranking function $R_{f 2}$ for TrBFNs can also be defined as, $R_{f 2}(A)=m(I) \sigma(I)-m(K) \sigma(K)=\frac{\left(t_{1}+t_{2}+t_{3}+t_{4}\right)\left(-t_{1}-t_{2}+t_{3}+t_{4}\right)}{8}-\frac{\left(k_{1}+k_{2}+k_{3}+k_{4}\right)\left(-k_{1}-k_{2}+k_{3}+k_{4}\right)}{8}$

Definition 2.4. Akram and Arshad [1].

Ranking Function $R_{f 1}$ for $T B F N$

Let $A=\langle I, K\rangle=\left\langle\left(t_{1}, t_{2}, t_{3}\right),\left(k_{1}, k_{2}, k_{3}\right)\right\rangle$ be the TBFN. Then by using Definition 2.2 , they have derived the ranking function $R_{f 1}$ of TBFN as $R_{f}(A)=(m(I)+\sigma(I)-(m(K)+\sigma(K)))$, where the means $(m(I), m(K))$ are defined as $m(I)=\frac{t_{1}+t_{2}+t_{3}}{3}, m(K)=\frac{k_{1}+k_{2}+k_{3}}{3}$ and the areas $(\sigma(I), \sigma(K))$ are defined as $\sigma(I)=\frac{t_{3}-t_{1}}{2}, \sigma(K)=\frac{k_{3}-k_{1}}{2}$.

Therefore, $R_{f 1}(A)=\frac{\left(-t_{1}+2 t_{2}+5 t_{3}\right)-\left(-k_{1}+2 k_{2}+5 k_{3}\right)}{6}=\frac{-t_{1}+2 t_{2}+5 t_{3}+k_{1}-2 k_{2}-5 k_{3}}{6}$.

If $\sigma(I), \sigma(K) \geq 1$, then the ranking function $R_{f 2}$ for TBFNs can also be defined as, $R_{f 2}(A)=m(I) \sigma(I)-m(K) \sigma(K)=\frac{\left(t_{1}+t_{2}+t_{3}\right)\left(t_{3}-t_{1}\right)}{6}-\frac{\left(k_{1}+k_{2}+k_{3}\right)\left(k_{3}-k_{1}\right)}{6}$

Definition 2.5. Ranking of Bipolar Fuzzy Numbers. Akram and Arshad [1].

Let $H=\left\{h_{1}, h_{2}, h_{3}, \ldots, h_{n}\right\}$ be the set of BFNs then for any distinct $h_{i}, h_{j} \in H$, the ranking function $R_{f 1}$ from $H$ to real line $\Re$ is mapping satisfying the following characteristics,

- If $R_{f 1}\left(h_{i}\right)<R_{f 1}\left(h_{j}\right)$, then $h_{i}<h_{j}$,

- If $R_{f 1}\left(h_{i}\right)=R_{f 1}\left(h_{j}\right)$, then $h_{i}=h_{j}$,

- If $R_{f 1}\left(h_{i}\right)>R_{f 1}\left(h_{j}\right)$, then $h_{i}>h_{j}$,

The ranking function for a $B F N h_{i}=\left\langle I_{i}, K_{i}\right\rangle=\left\langle\left(t_{i 1}, t_{i 2}, t_{i 3}, t_{i 4}\right),\left(k_{i 1}, k_{i 2}, k_{i 3}, k_{i 4}\right)\right\rangle$ as, $R_{f 1}\left(h_{i}\right)=\left[m\left(I_{i}\right)+\sigma\left(I_{i}\right)\right]-\left[m\left(K_{i}\right)+\sigma\left(K_{i}\right)\right], k=1,2,3,4$. Where $m\left(I_{i}\right)$ and $m\left(K_{i}\right)$ denote the mean of $I_{i}$ and the mean of $K_{i}$ respectively, $\sigma\left(I_{i}\right)$ represents the area of $I_{i}$ and $\sigma\left(K_{i}\right)$ represents the area of $K_{i}$.

If $\sigma\left(I_{i}\right), \sigma\left(K_{i}\right) \geq 1$ for each $i$, then the ranking function $R_{f 2}$ for TrBFNs can also be defined as,

$R_{f 2}\left(h_{i}\right)=m\left(I_{i}\right) \sigma\left(I_{i}\right)-m\left(K_{i}\right) \sigma\left(K_{i}\right)$

\section{Limitations of the Ranking Functions $R_{f 1}, R_{f 2}$ in ranking Trapezoidal Bipolar Fuzzy Numbers}

In this section, we discuss some limitations of Akram and Arshad's [1] ranking function in comparing different Trapezoidal Bipolar Fuzzy Numbers using numerical examples.

Definition 3.1. Let $A=\left\langle I_{1}, K_{1}\right\rangle=\left\langle\left(t_{11}, t_{12}, t_{13}, t_{14}\right),\left(k_{11}, k_{12}, k_{13}, k_{14}\right)\right\rangle$ and $B=\left\langle I_{2}, K_{2}\right\rangle=\left\langle\left(t_{21}, t_{22}, t_{23}, t_{24}\right),\left(k_{21}, k_{22}, k_{23}, k_{24}\right)\right\rangle$ be any two TrBFN. Using Definition 2.3, we can rewrite the Akram and Arshad's [1] ranking principle (Definition 2.5) that utilizes $R_{f 1}$ and $R_{f 2}$ in the following way, 
1. If $\frac{-t_{11}-t_{12}+3 t_{13}+3 t_{14}+k_{11}+k_{12}-3 k_{13}-3 k_{14}}{4}<\frac{-t_{21}-t_{22}+3 t_{23}+3 t_{24}+k_{21}+k_{22}-3 k_{23}-3 k_{24}}{4}$, then $A<B$.

2. If $\frac{-t_{11}-t_{12}+3 t_{13}+3 t_{14}+k_{11}+k_{12}-3 k_{13}-3 k_{14}}{4}=\frac{-t_{21}-t_{22}+3 t_{23}+3 t_{24}+k_{21}+k_{22}-3 k_{23}-3 k_{24}}{4}$, then $A=B$.

3. If $\frac{-t_{11}-t_{12}+3 t_{13}+3 t_{14}+k_{11}+k_{12}-3 k_{13}-3 k_{14}}{4}>\frac{-t_{21}-t_{22}+3 t_{23}+3 t_{24}+k_{21}+k_{22}-3 k_{23}-3 k_{24}}{4}$, then $A>B$.

4. If $\sigma\left(I_{i}\right), \sigma\left(K_{i}\right) \geq 1$, for each $i$, then the ranking function $R_{f 2}$ for TrBFNs can also be defined as,

$\frac{\left(t_{11}+t_{12}+t_{13}+t_{14}\right)\left(-t_{11}-t_{12}+t_{13}+t_{14}\right)-\left(k_{11}+k_{12}+k_{13}+k_{14}\right)\left(-k_{11}-k_{12}+k_{13}+k_{14}\right)}{8}$

The limitations of Akram and Arshad's [1] Ranking function can be seen from the following Examples.

Example 3.1. Let $A=\left\langle I_{1}, K_{1}\right\rangle=\langle(10,20,30,40),(10,20,30,40)\rangle$,

$B=\left\langle I_{2}, K_{2}\right\rangle=\langle(90,120,150,180),(90,120,150,180)\rangle$,

$C=\left\langle I_{3}, K_{3}\right\rangle=\langle(0,0,1,1),(0,0,1,1)\rangle$,

$D=\left\langle I_{4}, K_{4}\right\rangle=\langle(20,30,40,50),(20,30,40,50)\rangle$,

$E=\left\langle I_{5}, K_{5}\right\rangle=\langle(30,50,70,90),(30,50,70,90)\rangle$ be five different TrBFNs and their pictorial representation is given in Figure 1.

If we apply Akram and Arshad's [1] ranking function $R_{f}$ to the above five differ-

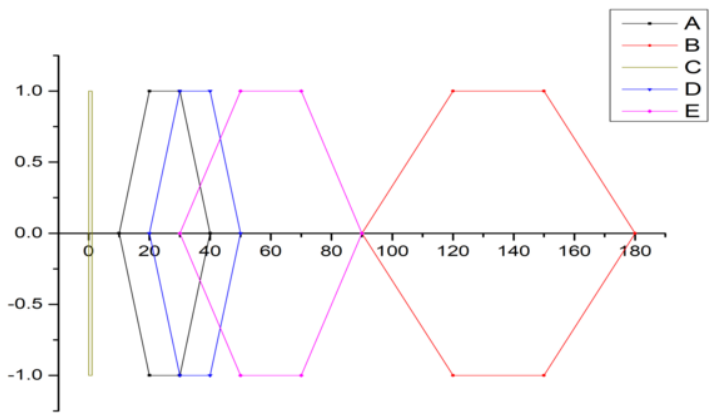

Figure 1. Pictorial representation of Example 3.1

ent TrBFNs, then we get $R_{f 1}(A)=R_{f 1}(B)=R_{f 1}(C)=R_{f 1}(D)=R_{f 1}(E)=0$ and $R_{f 2}(A)=R_{f 2}(B)=R_{f 2}(C)=R_{f 2}(D)=R_{f 2}(E)=0$ which implies that (from Definition 3.1) $A=B=C=D=E$. But from Figure 1, it is very clear that all the given TrBFNs are different in nature and which does not favor the human intuition.

Example 3.2. Let $A=\left\langle I_{1}, K_{1}\right\rangle=\langle(0.10,0.20,0.30,0.40),(0.50,0.60,0.70,0.80)\rangle$, $B=\left\langle I_{2}, K_{2}\right\rangle=\langle(0.3,0.4,0.5,0.6),(0.7,0.8,0.9,1)\rangle$, be two different TrBFNs and their pictorial representation is given in Figure 2.

If we apply Akram and Arshad's [1] ranking function $R_{f}$ to the above two different TrBFNs, then we get $R_{f 1}(A)=R_{f 1}(B)=-0.4$ which implies that (from Definition 3.1) $A=B$. This is an anti-intuitive case of Akram and Arshad's [1] ranking function. 


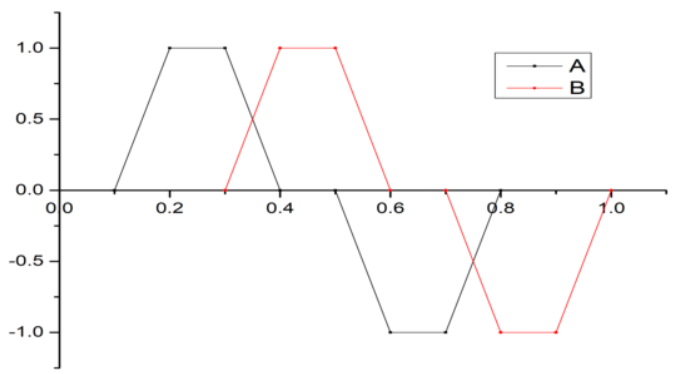

Figure 2. Pictorial representation of Example 3.2

Example 3.3. Let $A=\left\langle I_{1}, K_{1}\right\rangle=\langle(2,4,6,8),(4,5,6,7)\rangle$,

$B=\left\langle I_{2}, K_{2}\right\rangle=\langle(4,6,8,10),(6,7,8,9)\rangle$,

$C=\left\langle I_{3}, K_{3}\right\rangle=\langle(82,84,86,88),(84,85,86,87)\rangle$, be three different $\operatorname{Tr} B F N$ s and their pictorial representation is given in Figure 3.

If we apply Akram and Arshad's [1] ranking function $R_{f}$ to the above three differ-

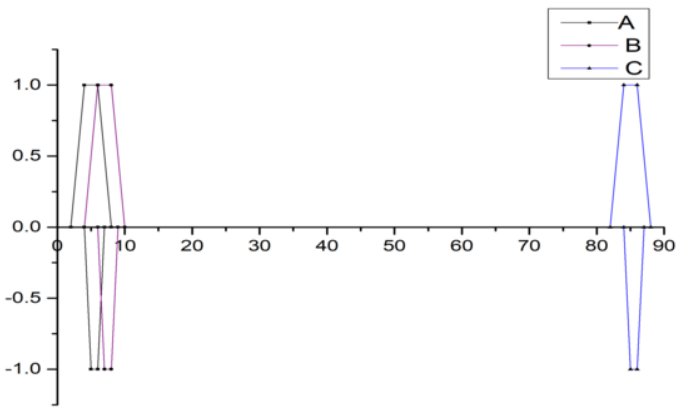

Figure 3. Pictorial representation of Example 3.3

ent TrBFNs, then we get $R_{f 1}(A)=R_{f 1}(B)=R_{f 1}(C)=1.5 \Rightarrow A=B=C$. Suppose if we consider the fourth formula (in Definition 3.1) then we get $R_{f 2}(A)=9, R_{f 2}(B)=$ $13, R_{f 2}(C)=169$ (Since $\sigma\left(I_{1}\right), \sigma\left(I_{2}\right), \sigma\left(I_{3}\right), \sigma\left(K_{1}\right), \sigma\left(K_{2}\right), \sigma\left(K_{3}\right)>1$ ) which implies that $A<B<C$. i.e., If we consider the second score function $R_{f 2}$ (once after the first score function $R_{f 1}$ fails), then we can rank the arbitrary TrBFNs effectively.

Example 3.4. Let $A=\left\langle I_{1}, K_{1}\right\rangle=\langle(5,20,30,40),(25,35,45,60)\rangle$,

$B=\left\langle I_{2}, K_{2}\right\rangle=\langle(10,15,25,45),(30,30,40,65)\rangle$,

$C=\left\langle I_{3}, K_{3}\right\rangle=\langle(0,25,25,45),(30,30,35,70)\rangle$,

$D=\left\langle I_{4}, K_{4}\right\rangle=\langle(12,13,13,57),(15,45,50,55)\rangle$,

$E=\left\langle I_{5}, K_{5}\right\rangle=\langle(2,23,27,43),(27,33,43,62)\rangle$ be five different TrBFNs and their pictorial representation is given in Figure 4.

If we apply Akram and Arshad's [1] ranking function $R_{f}$ to the above five different TrBFNs, then we get $R_{f 1}(A)=R_{f 1}(B)=R_{f 1}(C)=R_{f 1}(D)=R_{f 1}(E)=-17.5$ which implies that (from Definition 3.1) $A=B=C$. Suppose if we consider the fourth formula (in 


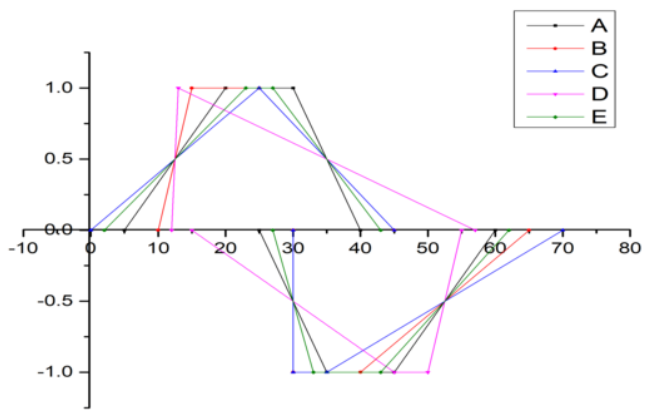

Figure 4. Pictorial representation of Example 3.4

Definition 3.1), then we get $R_{f 2}(A)=R_{f 2}(B)=R_{f 2}(C)=R_{f 2}(D)=R_{f 2}(E)=-393.75$ (Since $\sigma\left(I_{i}\right), \sigma\left(K_{i}\right)>1$, for $i=1$ to 5 ) which implies that $A=B=C=D=E$. i.e., In both the cases, ranking is same and it is anti-intuitive.

Example 3.5. Let $A=\left\langle I_{1}, K_{1}\right\rangle=\langle(5,70,90,110),(70,80,110,140)\rangle$, $B=\left\langle I_{2}, K_{2}\right\rangle=\langle(10,65,70,130),(60,90,120,130)\rangle$,

$C=\left\langle I_{3}, K_{3}\right\rangle=\langle(20,55,75,125),(40,110,115,135)\rangle$, be three different TrBFNs and their pictorial representation is given in Figure 5.

If we apply Akram and Arshad's [1] ranking function $R_{f}$ to the above five different

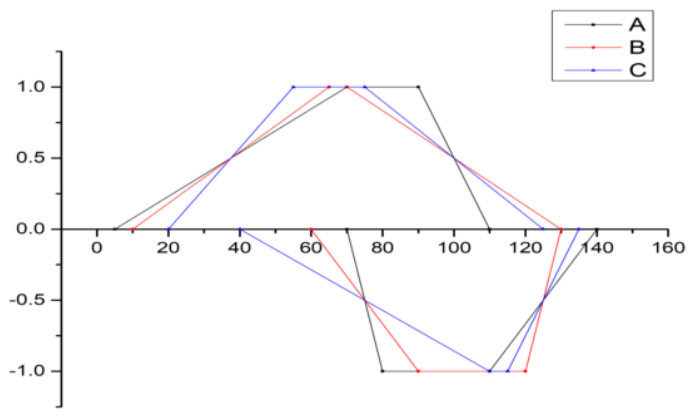

Figure 5. Pictorial representation of Example 3.5

TrBFNs, then we get $R_{f 1}(A)=R_{f 1}(B)=R_{f 1}(C)=-18.75$ which implies that (from Definition 3.1) $A=B=C$. Suppose if we consider the fourth formula (in Definition 3.1), then we get $R_{f 2}(A)=R_{f 2}(B)=R_{f 2}(C)=-703.125$ (Since $\sigma\left(I_{i}\right), \sigma\left(K_{i}\right)>1$, for $i=$ 1 to 3$)$ which implies that $A=B=C$. i.e., In both the cases, ranking is same and it is anti-intuitive.

\subsection{Limitations of Ranking functions $R_{f 1}, R_{f 2}$ of Triangular Bipolar Fuzzy Numbers}

In this subsection, we discuss the various drawbacks of Akram and Arshad's [1] ranking function in comparing different Triangular Bipolar Fuzzy Numbers using numerical examples. 
Definition 3.2. Let $A=\left\langle I_{1}, K_{1}\right\rangle=\left\langle\left(t_{11}, t_{12}, t_{13}\right),\left(k_{11}, k_{12}, k_{13}\right)\right\rangle$ and $B=\left\langle I_{2}, K_{2}\right\rangle=\left\langle\left(t_{21}, t_{22}, t_{23}\right),\left(k_{21}, k_{22}, k_{23}\right)\right\rangle$ be any two TrBFN. Using Definition 2.4 , we can rewrite the Akram and Arshad's [1] ranking principle (Definition 2.5) in the following way,

1. If $\frac{-t_{11}+2 t_{12}+5 t_{13}+k_{11}-2 k_{12}-5 k_{13}}{6}<\frac{-t_{21}+2 t_{22}+5 t_{23}+k_{21}-2 k_{22}-5 k_{23}}{6}$, then $A<B$.

2. If $\frac{-t_{11}+2 t_{12}+5 t_{13}+k_{11}-2 k_{12}-5 k_{13}}{6}=\frac{-t_{21}+2 t_{22}+5 t_{23}+k_{21}-2 k_{22}-5 k_{23}}{6}$, then $A=B$.

3. If $\frac{-t_{11}+2 t_{12}+5 t_{13}+k_{11}-2 k_{12}-5 k_{13}}{6}>\frac{-t_{21}+2 t_{22}+5 t_{23}+k_{21}-2 k_{22}-5 k_{23}}{6}$, then $A>B$.

4. If $\sigma\left(I_{i}\right), \sigma\left(K_{i}\right) \geq 1$, for each $i$, then the ranking function $R_{f 2}$ for TBFNs can also be defined as, $\frac{\left(t_{11}+t_{12}+t_{13}\right)\left(t_{13}-t_{11}\right)-\left(k_{11}+k_{12}+k_{13}\right)\left(k_{13}-k_{11}\right)}{6}$

Akram and Arshad [1] have introduced the ranking method without investigating its Mathematical Properties. Ranking principle of TBFN defined in [1] is not true for any two TBFNs which can be seen by using the following examples,

Example 3.6. Let $A=\left\langle I_{1}, K_{1}\right\rangle=\langle(3,4.2,4.8),(4.2,5.1,6)\rangle$,

$B=\left\langle I_{2}, K_{2}\right\rangle=\langle(3.6,4.8,4.92),(4.8,5.7,6.12)\rangle$,

$C=\left\langle I_{3}, K_{3}\right\rangle=\langle(4.8,5.1,5.16),(6,6,6.36)\rangle$, be three different TBFNs and their pictorial representation is given in Figure 6.

If we apply Akram and Arshad's [1] ranking function $R_{f}$ to the above 3 different

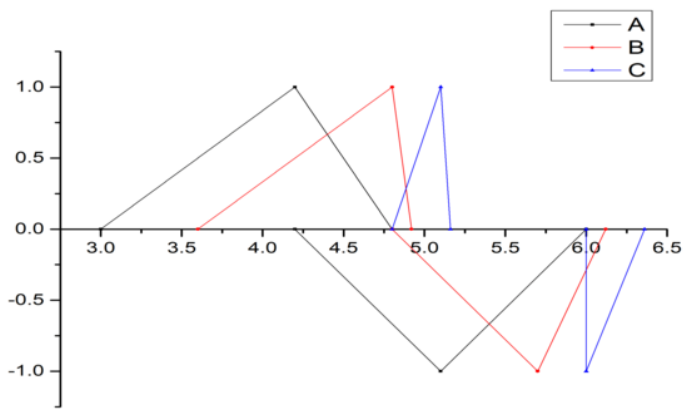

Figure 6. Pictorial representation of Example 3.6

TBFNs, then we get $R_{f 1}(A)=R_{f 1}(B)=R_{f 1}(C)=-1.1, R_{f 2}(A)=-0.99, R_{f 2}(B)=$ $-0.726, R_{f 2}(C)=-0.198$ which implies that (from Definition 3.2) $A<B<C$. i.e., If we consider the second score function $R_{f 2}$ (once after the first score function $R_{f 1}$ fails), then we can rank the arbitrary TBFNs effectively.

Example 3.7. Let $A=\left\langle I_{1}, K_{1}\right\rangle=\langle(30,50,60),(30,50,60)\rangle, B=\left\langle I_{2}, K_{2}\right\rangle=\langle(40,60,70)$, $(40,60,70)\rangle, C=\left\langle I_{3}, K_{3}\right\rangle=\langle(10,20,30),(10,20,30)\rangle, D=\left\langle I_{4}, K_{4}\right\rangle=\langle(1,2,3),(1,2,3)\rangle$, $E=\left\langle I_{5}, K_{5}\right\rangle=\langle(50,70,90),(50,70,90)\rangle, F=\left\langle I_{6}, K_{6}\right\rangle=\langle(20,50,70),(20,50,70)\rangle$ be six different TBFNs and their pictorial representation is given in Figure 7. If we apply Akram and Arshad's [1] ranking function $R_{f}$ to the above five different 


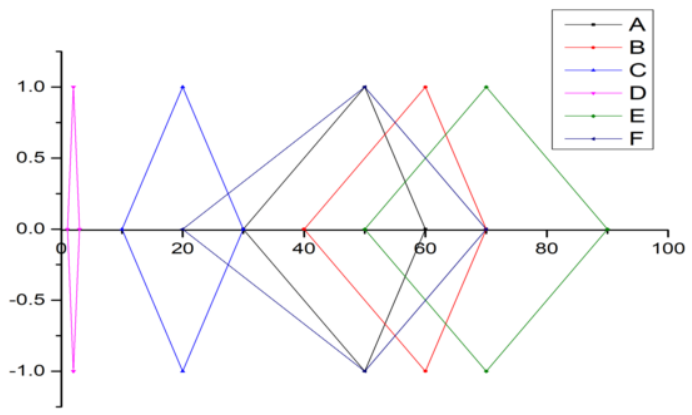

Figure 7. Pictorial representation of Example 3.7

TBFNs, then we get $R_{f 1}(A)=R_{f 1}(B)=R_{f 1}(C)=R_{f 1}(D)=R_{f 1}(E)=R_{f 1}(F)=0$ which implies that (from Definition 3.2) $A=B=C=D=E=F$. Suppose if we consider the fourth formula (in Definition 3.2), then we get $R_{f 2}(A)=R_{f 2}(B)=R_{f 2}(C)=$ $R_{f 2}(D)=R_{f 2}(E)=R_{f 2}(F)=0\left(\right.$ Since $\sigma\left(I_{i}\right), \sigma\left(K_{i}\right)>1$, for $i=1$ to 6) which implies that $A=B=C=D=E=F$. i.e., In both the cases, ranking is same and it does not favor the human intuition.

\subsection{Limitations of $R_{f}$}

In this subsection, we discuss some limitations of the score functions $R_{f 1}$ and $R_{f 2}$ in a Mathematical way.

1. Let $A_{1}=\left\langle I_{1}, K_{1}\right\rangle=\left\langle\left(t_{11}, t_{12}, t_{13}, t_{14}\right),\left(t_{11}, t_{12}, t_{13}, t_{14}\right)\right\rangle, A_{2}=\left\langle I_{2}, K_{2}\right\rangle=\left\langle\left(t_{21}, t_{22}\right.\right.$, $\left.\left.t_{23}, t_{12}\right),\left(t_{21}, t_{22}, t_{23}, t_{24}\right)\right\rangle$ any two TrBFNs. Then $R_{f 1}\left(A_{1}\right)=R_{f 1}\left(A_{2}\right)=0$ and $R_{f 2}\left(A_{1}\right)=R_{f 2}\left(A_{2}\right)=0$. (Example 3.1 represents the numerical illustration of this Theoretical (Mathematical) drawback 1)

2. Let $A_{1}=\left\langle I_{1}, K_{1}\right\rangle=\left\langle\left(t_{11}, t_{12}, t_{13}, t_{14}\right),\left(k_{11}, k_{12}, k_{13}, k_{14}\right)\right\rangle, A_{2}=\left\langle I_{2}, K_{2}\right\rangle=\left\langle\left(t_{11}+\right.\right.$ $\left.\left.\varepsilon, t_{12}+\varepsilon, t_{13}+\varepsilon, t_{14}+\varepsilon\right),\left(k_{11}+\varepsilon, k_{12}+\varepsilon, k_{13}+\varepsilon, k_{14}+\varepsilon\right)\right\rangle$ any two TrBFNs and $\varepsilon \leq t_{11}, k_{11}$. Then $R_{f 1}\left(A_{1}\right)=R_{f 1}\left(A_{2}\right)$. (Example 3.2, Example 3.3 represent the numerical illustration of this Theoretical (Mathematical) drawback)

3. Let $A_{1}=\left\langle I_{1}, K_{1}\right\rangle=\left\langle\left(t_{11}, t_{12}, t_{13}, t_{14}\right),\left(k_{11}, k_{12}, k_{13}, k_{14}\right)\right\rangle, A_{2}=\left\langle I_{2}, K_{2}\right\rangle=\left\langle\left(t_{11}-\right.\right.$ $\left.\left.\varepsilon_{1}, t_{12}+\varepsilon_{2}, t_{13}-\varepsilon_{1}, t_{14}+\varepsilon_{2}\right),\left(k_{11}+\varepsilon_{1}, k_{12}-\varepsilon_{2}, k_{13}+\varepsilon_{1}, k_{14}-\varepsilon_{2}\right)\right\rangle$ any two TrBFNs and $\varepsilon_{1} \leq t_{11}, \varepsilon_{2} \leq k_{12}$ and $\varepsilon_{1} \leq \varepsilon_{2}$. Then $R_{f 1}\left(A_{1}\right)=R_{f 1}\left(A_{2}\right)$.

4. Let $A_{1}=\left\langle I_{1}, K_{1}\right\rangle=\left\langle\left(t_{11}, t_{12}, t_{13}, t_{14}\right),\left(k_{11}, k_{12}, k_{13}, k_{14}\right)\right\rangle, A_{2}=\left\langle I_{2}, K_{2}\right\rangle=\left\langle\left(t_{11}-\right.\right.$ $\left.\left.\varepsilon, t_{12}+\varepsilon, t_{13}-\varepsilon, t_{14}+\varepsilon\right),\left(k_{11}+\varepsilon, k_{12}-\varepsilon, k_{13}+\varepsilon, k_{14}-\varepsilon\right)\right\rangle$ any two TrBFNs and $\varepsilon \leq t_{11}, k_{11}$. Then $R_{f 1}\left(A_{1}\right)=R_{f 1}\left(A_{2}\right)$ and $R_{f 2}\left(A_{1}\right)=R_{f 2}\left(A_{2}\right)$.

5. Let $A_{1}=\left\langle I_{1}, K_{1}\right\rangle=\left\langle\left(t_{11}, t_{12}, t_{13}, t_{14}\right),\left(k_{11}, k_{12}, k_{13}, k_{14}\right)\right\rangle, A_{2}=\left\langle I_{2}, K_{2}\right\rangle=\left\langle\left(t_{11}-\right.\right.$ $\left.\left.\varepsilon, t_{12}+\varepsilon, t_{13}+\varepsilon, t_{14}-\varepsilon\right),\left(k_{11}-\varepsilon, k_{12}+\varepsilon, k_{13}+\varepsilon, k_{14}-\varepsilon\right)\right\rangle$ any two TrBFNs and $\varepsilon \leq t_{11}, k_{11}$. Then $R_{f 1}\left(A_{1}\right)=R_{f 1}\left(A_{2}\right)$ and $R_{f 2}\left(A_{1}\right)=R_{f 2}\left(A_{2}\right)$.

Note: Here, all the values of $\varepsilon_{i}$ have been chosen without violating the conditions of TrBFNs (TBFNs). Numerical illustration of these theoretical drawbacks 3, 4, and 5 are given in the example 3.4, example 3.5, example 3.6 and example 3.7. 
From the limitations of Akram and Arshad's [1] score functions, we can conclude that the score functions $R_{f 1}$ and $R_{f 2}$ are not sufficient for ranking any two TRBFNs (TBFNs). In order to improve the ranking principle, we define a new score function in the following section.

\section{New Score function $R_{f 3}$}

In this section, first, we define a new score function on the class of TrBFNs (TBFNs) that can overcome the drawbacks of Akram and Arshad's ranking functions. Then we study some properties of the proposed score function, and finally, we discuss the efficiency of a score function in ranking TrBFNs that are not ranked by using $R_{f 1}$ and $R_{f 2}$. From the previous limitations, we can conclude that if the mean and area of both the membership and non-membership functions of any two TrBFNs are equal, then they are ranked equal. In order to distinguish any two TrBFNs with same mean and area, here we made a small change in the score function $R_{f}$ and defined a new score function $R_{f 3}$ on the class of Trapezoidal Bipolar Fuzzy Numbers as follows.

Definition 4.1. Ranking Function $R_{3 f}$ for TrBFN

Let $A=\langle I, K\rangle=\left\langle\left(t_{1}, t_{2}, t_{3}, t_{4}\right),\left(k_{1}, k_{2}, k_{3}, k_{4}\right)\right\rangle$ be any $\operatorname{Tr} B F N$. Then the new score function $R_{f 3}$ of TrBFN is defined as $R_{3 f}(A)=\frac{\left(m(I)+\frac{-t_{1}-t_{2}+t_{3}+t_{4}}{2}\right) t_{4}+\left(m(K)+\frac{-k_{1}-k_{2}+k_{3}+k_{4}}{2}\right) k_{4}}{1+m(I)+m(K)}$, where $m(I)=\frac{t_{1}+t_{2}+t_{3}+t_{4}}{4}$ and $m(K)=\frac{k_{1}+k_{2}+k_{3}+k_{4}}{4}$.

Therefore, $R_{f 3}(A)=\frac{\left(-t_{1}-t_{2}+3 t_{3}+3 t_{4}\right) t_{4}+\left(-k_{1}-k_{2}+3 k_{3}+3 k_{4}\right) k_{4}}{4+t_{1}+t_{2}+t_{3}+t_{4}+k_{1}+k_{2}+k_{3}+k_{4}}$.

Definition 4.2. Ranking Principle on the class of $\operatorname{Tr} B F N(T B F N)$ :

Let $A=\left\{A_{1}, A_{2}, \ldots, A_{n}\right\}$ be the set of TrBFNs (TBFNs) then for any distinct $A_{i}, A_{j} \in A$, the ranking functions $R_{f i}, i=1,2,3$ from $A$ to real line $\mathfrak{R}$ is a mapping satisfying the following characteristics,

- If $R_{f 1}\left(A_{i}\right)<R_{f 1}\left(A_{j}\right)$, then $A_{i}<A_{j}$

- If $R_{f 1}\left(A_{i}\right)>R_{f 1}\left(A_{j}\right)$, then $A_{i}>A_{j}$ or

- If $R_{f 1}\left(A_{i}\right)=R_{f 1}\left(A_{j}\right)$, then

* If $R_{f 2}\left(A_{i}\right)<R_{f 2}\left(A_{j}\right)$, then $A_{i}<A_{j}$

* If $R_{f 2}\left(A_{i}\right)>R_{f 2}\left(A_{j}\right)$, then $A_{i}>A_{j}$ or

* If $R_{f 1}\left(A_{i}\right)=R_{f 1}\left(A_{j}\right), R_{f 2}\left(A_{i}\right)=R_{f 2}\left(A_{j}\right)$, then

* If $R_{f 3}\left(A_{i}\right)<R_{f 3}\left(A_{j}\right)$, then $A_{i}<A_{j}$

* If $R_{f 3}\left(A_{i}\right)>R_{f 3}\left(A_{j}\right)$, then $A_{i}>A_{j}$ or

* If $R_{f 1}\left(A_{i}\right)=R_{f 1}\left(A_{j}\right), R_{f 2}\left(A_{i}\right)=R_{f 2}\left(A_{j}\right)$ and $R_{f 3}\left(A_{i}\right)=R_{f 3}\left(A_{j}\right)$, then $A_{i} \approx$ $A_{j}$.

\subsection{Properties of $R_{f 1}, R_{f 2}, R_{f 3}$ and their comparison}

In this subsection, we see the properties of different ranking functions $R_{f 1}, R_{f 2}, R_{f 3}$. 
1. The function $R_{f}$ maps the set of TrBFNs (which are Symmetric about X-axis) to 0

Let $A=\left\{A_{i} \mid A_{i}=\left\langle I_{i}, K_{i}\right\rangle=\left\langle\left(t_{i 1}, t_{i 2}, t_{i 3}, t_{i 4}\right),\left(t_{i 1}, t_{i 2}, t_{i 3}, t_{i 4}\right)\right\rangle\right\}$ the set of TrBFNs. Then $R_{f 1}\left(A_{i}\right)=0$ and $R_{f 2}\left(A_{i}\right)=0$. This property is true for any TBFN. But $R_{f 3}$ is not mapping the set of TrBFNs (TBFNs) (that are symmetric about $\mathrm{X}$-axis) to zero.

2. Let $A_{1}=\left\langle I_{1}, K_{1}\right\rangle=\left\langle\left(t_{1}, t_{2}, t_{3}\right),\left(k_{1}, k_{2}, k_{3}\right)\right\rangle$ be any Triangular Bipolar fuzzy number (TBFN). Then $R_{f 3}(A)=\frac{\left(m(I)+\frac{t_{3}-t_{1}}{2}\right) t_{3}+\left(m(K)+\frac{k_{3}-k_{1}}{2}\right) k_{3}}{1+m(I)+m(K)}$

$=\frac{\left(\frac{t_{1}+t_{2}+t_{3}}{3}+\frac{t_{3}-t_{1}}{2}\right) t_{3}+\left(\frac{k_{1}+k_{2}+k_{3}}{3}+\frac{k_{3}-k_{1}}{2}\right) k_{3}}{1+\frac{t_{1}+t_{2}+t_{3}}{3}+\frac{k_{1}+k_{2}+k_{3}}{3}}=\frac{\left(\frac{-t_{1}+2 t_{2}+5 t_{3}}{6}\right) t_{3}+\left(\frac{-k_{1}+2 k_{2}+5 k_{3}}{6}\right) k_{3}}{\frac{\left(3+t_{1}+t_{2}+t_{3}+k_{1}+k_{2}+k_{3}\right)}{3}}$

$=\frac{\left(-t_{1}+2 t_{2}+5 t_{3}\right) t_{3}+\left(-k_{1}+2 k_{2}+5 k_{3}\right) k_{3}}{2\left(3+t_{1}+t_{2}+t_{3}+k_{1}+k_{2}+k_{3}\right)}$.

3. Ranking function $R_{f 1}$ is Translation Invariant:

Let $A_{1}=\left\langle I_{1}, K_{1}\right\rangle=\left\langle\left(t_{11}, t_{12}, t_{13}, t_{14}\right),\left(k_{11}, k_{12}, k_{13}, k_{14}\right)\right\rangle, A_{2}=\left\langle I_{2}, K_{2}\right\rangle=\left\langle\left(t_{11}+\right.\right.$ $\left.\left.\varepsilon, t_{12}+\varepsilon, t_{13}+\varepsilon, t_{14}+\varepsilon\right),\left(k_{11}+\varepsilon, k_{12}+\varepsilon, k_{13}+\varepsilon, k_{14}+\varepsilon\right)\right\rangle$ any two TrBFNs. Then $R_{f 1}\left(A_{1}\right)=R_{f 1}\left(A_{2}\right)$.

i.e., $R_{f}(A+\varepsilon)=R_{f}(A), \forall \varepsilon \geq 0$. This property holds for the set of TBFNs too. $R_{f 2}$ and $R_{f 3}$ are not Translation Invariant.

4. Let $\left.A_{1}=\left\langle I_{1}, K_{1}\right\rangle=\left\langle\left[t_{1}, t_{2}\right],\left[k_{1}, k_{2}\right]\right)\right\rangle$ be any interval-valued Bipolar fuzzy number (IVBFN). Then $R_{f 1}(A)=t_{2}-k_{2}, R_{f 2}(A)=\frac{t_{2}{ }^{2}-t_{1}{ }^{2}-k_{2}{ }^{2}+k_{1}{ }^{2}}{4}$ and $R_{f 3}(A)=$ $\frac{2\left(t_{2}^{2}+k_{2}^{2}\right)}{2+t_{1}+t_{2}+k_{1}+k_{2}}$.

Observation: For the interval-valued BFNs,

- The score function $R_{f 1}$ represents the length between the supremum of membership function and supremum of the non-membership function. $R_{f 1}$ does not consider the other two legs $t_{1}, k_{1}$ in the score function which is a limitation for the score function $R_{f 1}$. But the score function $R_{f 2}$ overcomes this limitation.

- The score function $R_{f 2}$ has the drawback that, if $t_{1}=k_{1}, t_{2}=k_{2}$, then $R_{f 2}(A)=$ 0 . However, this drawback has been overcome by $R_{f 3}$. In this way, a sequential ordering in the Definition 4.2 performs better.

5. - For any $A_{1}=\left\langle I_{1}, K_{1}\right\rangle=\left\langle\left(t_{11}, t_{12}, t_{13}, t_{14}\right),\left(k_{11}, k_{12}, k_{13}, k_{14}\right)\right\rangle \operatorname{TrBFN}$ (where all $t_{1 k}, k_{1 k} \in[0,1], k=1,2,3,4$.), $R_{f 1}(A) \in\left[-\frac{3}{2}, \frac{3}{2}\right]$.

- If $A=\langle(0,0,0,0),(0,0,1,1)\rangle$, then $R_{f 1}(A)=-\frac{3}{2}$.

- If $A=\langle(0,0,1,1),(0,0,0,0)\rangle$, then $R_{f 1}(A)=\frac{3}{2}$.

Definition 4.3. Let $\zeta=\left\{A_{i} \mid A_{i}=\left\langle\left(t_{i 1}, t_{i 2}, t_{i 3}, t_{i 4}\right),\left(k_{i 1}, k_{i 2}, k_{i 3}, k_{i 4}\right)\right\rangle\right\}$ be the subset of the set of TrBFNs. Then the subset relation $\subseteq$ is defined as, $A_{1} \subseteq A_{2}$, if $t_{11} \geq t_{21}, t_{12} \geq$ $t_{22}, t_{13} \leq t_{23}, t_{14} \leq t_{24}$ and $k_{11} \leq k_{21}, k_{12} \leq k_{22}, k_{13} \geq k_{23}, k_{14} \geq k_{24}$.

From the above properties and the limitations, we can conclude that Arshad and Akram's ranking principle alone cannot discriminate any two TrBFNs (TBFNs). That is, Akram and Arshad's [1] ranking principle does not define Total ordering on the entire class of TrBFNs (TBFNs). However, Akram and Arshad's [1] ranking function $\left(R_{f}\right)$ can define Total ordering in the subset $\zeta$ which can be proved using the following theorem.

Theorem 4.1. Let $A_{1}, A_{2} \in \zeta$. If $A_{1} \subseteq A_{2}$, then $R_{f i}\left(A_{1}\right) \leq R_{f i}\left(A_{2}\right)$.

Proof: Case 1: If $A_{1} \subseteq A_{2}$, then $R_{f 1}\left(A_{1}\right) \leq R_{f 1}\left(A_{2}\right)$

We assume that $A_{1}, A_{2} \in \zeta$ and $A_{1} \subseteq A_{2}$ which imply that 


$$
t_{11} \geq t_{21}, t_{12} \geq t_{22}, t_{13} \leq t_{23}, t_{14} \leq t_{24} \text { and } k_{11} \leq k_{21}, k_{12} \leq k_{22}, k_{13} \geq k_{23}, k_{14} \geq k_{24}
$$

Therefore from equation 1, we get

$$
t_{11}+t_{12} \geq t_{21}+t_{22}, t_{13}+t_{14} \leq t_{23}+t_{24},
$$

and

$$
k_{11}+k_{12} \leq k_{21}+k_{22}, k_{13}+k_{14} \geq k_{23}+k_{24}
$$

By using Equation 2 and Equation 3, we get

$$
-t_{11}-t_{12}+3 t_{13}+3 t_{14} \leq-t_{21}-t_{22}+3 t_{23}+3 t_{24}
$$

and

$$
k_{11}+k_{12}-3 k_{13}-3 k_{14} \leq k_{21}+k_{22}-3 k_{23}-3 k_{24}
$$

By adding Equation 4 and Equation 5, we get

$-t_{11}-t_{12}+3 t_{13}+3 t_{14}+k_{11}+k_{12}-3 k_{13}-3 k_{14} \leq-t_{21}-t_{22}+3 t_{23}+3 t_{24}+k_{21}+k_{22}-3 k_{23}-3 k_{24}$

Dividing both the sides of Equation 6 by 4 , we get $R_{f 1}(A) \leq R_{f 1}(B)$ (See Definition 2.3). Hence the proof.

Note: Proofs of other cases are similar to case 1 and hence they are omitted.

The ranking principle given in Definition 4.2 is an updated version of the ranking principle given by Akram and Arshad's [1] (Definition 2.3, 3.1). Adding a new score function $R_{f 3}$ to the ranking principle in Definition 2.3 gives a better ranking scenario which can be seen from the following Table 1. 
Table 1. Efficiency of the proposed ranking function

\begin{tabular}{|c|c|c|}
\hline Examples & Akram and Arshad Ranking Principle & Proposed Ranking Principle \\
\hline $\begin{array}{l}\text { Let } A=\left\langle I_{1}, K_{1}\right\rangle=\langle(10,20,30,40),(10,20,30,40)\rangle \\
B=\left\langle I_{2}, K_{2}\right\rangle=\langle(90,120,150,180),(90,120,150,180)\rangle \\
C=\left\langle I_{3}, K_{3}\right\rangle=\langle(0,0,1,1),(0,0,1,1)\rangle \\
D=\left\langle I_{4}, K_{4}\right\rangle=\langle(20,30,40,50),(20,30,40,50)\rangle \\
E=\left\langle I_{5}, K_{5}\right\rangle=\langle(30,50,70,90),(30,50,70,90)\rangle \\
\text { be five different TrBFNs. }\end{array}$ & $\begin{array}{l}R_{f 1}(A)=R_{f 1}(B)=R_{f 1}(C)=R_{f 1}(D)=R_{f 1}(E)=0 \\
\text { and } R_{f 2}(A)=R_{f 2}(B)=R_{f 2}(C)=R_{f 2}(D)=R_{f 2}(E)=0 \\
\Rightarrow A=B=C=D=E\end{array}$ & $\begin{array}{l}R_{f 1}(A)=R_{f 1}(B)=R_{f 1}(C)=R_{f 1}(D)=R_{f 1}(E)=0 \\
, R_{f 2}(A)=R_{f 2}(B)=R_{f 2}(C)=R_{f 2}(D)=R_{f 2}(E)=0 \\
R_{f 3}(A)=17.64, R_{f 3}(B)=64.76, R_{f 3}(C)=0.375 \\
R_{f 3}(D)=19.36, R_{f 3}(E)=37.19 \\
\Rightarrow C<A<D<E<B\end{array}$ \\
\hline $\begin{array}{l}\text { Let } A=\left\langle I_{1}, K_{1}\right\rangle=\langle(0.1,0.2,0.3,0.4),(0.5,0.6,0.7,0.8)\rangle \\
B=\left\langle I_{2}, K_{2}\right\rangle=\langle(0.3,0.4,0.5,0.6),(0.7,0.8,0.9,1)\rangle \\
\text { be two different TrBFNs. }\end{array}$ & $\begin{array}{l}R_{f 1}(A)=R_{f 1}(B)=-0.4 \\
\text { and } R_{f 2}(A)=R_{f 2}(B)=-0.08 \Rightarrow A=B\end{array}$ & $\begin{array}{l}R_{f 1}(A)=R_{f 1}(B)=-0.4, R_{f 2}(A)=R_{f 2}(B)=-0.4 \\
\text { and } R_{f 3}(A)=0.113<R_{f 3}(B)=0.156 \Rightarrow A<B\end{array}$ \\
\hline $\begin{array}{l}\text { Let } A=\left\langle I_{1}, K_{1}\right\rangle=\langle(5,20,30,40),(25,35,45,60)\rangle \\
B=\left\langle I_{2}, K_{2}\right\rangle=\langle(10,15,25,45),(30,30,40,65)\rangle \\
C=\left\langle I_{3}, K_{3}\right\rangle=\langle(0,25,25,45),(30,30,35,70)\rangle \\
D=\left\langle I_{4}, K_{4}\right\rangle=\langle(12,13,13,57),(15,45,50,55)\rangle \\
E=\left\langle I_{5}, K_{5}\right\rangle=\langle(2,23,27,43),(27,33,43,62)\rangle \\
\text { be five different TrBFNs. }\end{array}$ & $\begin{array}{l}R_{f 1}(A)=R_{f 1}(B)=R_{f 1}(C)=R_{f 1}(D)=R_{f 1}(E)=-17.5 \\
R_{f 2}(A)=R_{f 2}(B)=R_{f 2}(C)=R_{f 2}(D)=R_{f 2}(E)=-393.75 \\
\Rightarrow A=B=C=D=E\end{array}$ & $\begin{array}{l}R_{f 1}(A)=R_{f 1}(B)=R_{f 1}(C)=R_{f 1}(D)=R_{f 1}(E)=-17.5 \\
R_{f 2}(A)=R_{f 2}(B)=R_{f 2}(C)=R_{f 2}(D)=R_{f 2}(E)=-393.75 \\
\text { and } R_{f 3}(A)=21.49, R_{f 3}(B)=23.57, R_{f 3}(C)=24.78 \\
R_{f 3}(D)=23.26, R_{f 3}(E)=22.50 \\
\text { RightarrowA }<E<D<B<C\end{array}$ \\
\hline $\begin{array}{l}\text { Let } A=\left\langle I_{1}, K_{1}\right\rangle=\langle(5,70,90,110),(70,80,110,140)\rangle, \\
B=\left\langle I_{2}, K_{2}\right\rangle=\langle(10,65,70,130),(60,90,120,130)\rangle \\
C=\left\langle I_{3}, K_{3}\right\rangle=\langle(20,55,75,125),(40,110,115,135)\rangle \\
\text { be three different TrBFNs. }\end{array}$ & $\begin{array}{l}R_{f 1}(A)=R_{f 1}(B)=R_{f 1}(C)=-18.75 \\
R_{f 2}(A)=R_{f 2}(B)=R_{f 2}(C)=-703.125 \\
\Rightarrow A=B=C\end{array}$ & $\begin{array}{l}R_{f 1}(A)=R_{f 1}(B)=R_{f 1}(C)=-18.75 \\
R_{f 2}(A)=R_{f 2}(B)=R_{f 2}(C)=-703.125, \text { and } \\
R_{f 3}(A)=52.19<R_{f 3}(B)=53.84<R_{f 3}(C)=53.98 \Rightarrow A<B<C\end{array}$ \\
\hline $\begin{array}{l}\text { Let } A=\left\langle I_{1}, K_{1}\right\rangle=\langle(30,50,60),(30,50,60)\rangle \\
B=\left\langle I_{2}, K_{2}\right\rangle=\langle(40,60,70),(40,60,70)\rangle \\
C=\left\langle I_{3}, K_{3}\right\rangle=\langle(10,20,30),(10,20,30)\rangle \\
D=\left\langle I_{4}, K_{4}\right\rangle=\langle(1,2,3),(1,2,3)\rangle \\
E=\left\langle I_{5}, K_{5}\right\rangle=\langle(50,70,90),(50,70,90)\rangle \\
F=\left\langle I_{6}, K_{6}\right\rangle=\langle(20,50,70),(20,50,70)\rangle \\
\text { be six different TBFNs. }\end{array}$ & $\begin{array}{l}R_{f 1}(A)=R_{f 1}(B)=R_{f 1}(C)=R_{f 1}(D)=R_{f 1}(E)=R_{f 1}(F)=0, \\
R_{f 2}(A)=R_{f 2}(B)=R_{f 2}(C)=R_{f 2}(D)=R_{f 2}(E)=R_{f 2}(F)=0\end{array}$ & $\begin{array}{l}R_{f 1}(A)=R_{f 1}(B)=R_{f 1}(C)=R_{f 1}(D)=R_{f 1}(E)=R_{f 1}(F)=0 \\
R_{f 2}(A)=R_{f 2}(B)=R_{f 2}(C)=R_{f 2}(D)=R_{f 2}(E)=R_{f 2}(F)=0 \\
\text { and } R_{f 3}(A)=78.44, R_{f 3}(B)=87.75, R_{f 3}(C)=43.90 \\
R_{f 3}(D)=3.6, R_{f 3}(E)=114.89, R_{f 3}(F)=106.36 \\
\Rightarrow D<C<A<B<F<E\end{array}$ \\
\hline
\end{tabular}




\section{Conclusion}

In this paper, the shortcomings of a new ranking function introduced by Akram and Arshad [1] were discussed using various cases. Further, We proposed a new ranking function and studied its properties. Finally, we introduced a new ranking principle by combining Akram and Arshad's [1] ranking function and the proposed ranking function and also the efficiency of the proposed ranking principle in comparing arbitrary TrBFNs discussed. The proposed ranking principle can give better results for the decision-making problems under Bipolar fuzzy environment, which would be studied in future.

\section{References}

[1] Akram, Muhammad and Arshad, Maham, (2019). A Novel Trapezoidal Bipolar Fuzzy TOPSIS Method for Group Decision-Making. Group Decision and Negotiation, 28, 565-584.

[2] Atanassov, K. T. (1986). Intuitionistic fuzzy sets. Fuzzy Sets and Systems, 20, 87-96.

[3] Atanassov, K. T., \& Gargov, G, (1989). Interval-valued intuitionistic fuzzy sets. Fuzzy Sets and Systems, 31(3), 343-349.

[4] Akram, M., Shumaiza and M. Arshad, (2020). Bipolar fuzzy TOPSIS and bipolar fuzzy ELECTRE-I methods to diagnosis. Computational and Applied Mathematics, 39, 1-21.

[5] Nayagam, VLG., Dhanasekaran, P. ,Jeevaraj, S. (2016). A complete ranking of incomplete trapezoidal information. Journal of Intelligent \& Fuzzy Systems 30 (6), 3209-3225.

[6] Dhanasekaran, P. ,Jeevaraj, S., Nayagam, VLG. (2018).A complete ranking of trapezoidal fuzzy numbers and its applications to multi-criteria decision making. Neural Computing and Applications, 30 (11), 3303-3315.

[7] Jeevaraj S (2021). Ordering of interval-valued Fermatean fuzzy sets and its applications. Expert Systems with Applications 185, 115613.

[8] Przemyslaw Grzegorzewski, Olgierd Hryniewicz, Maciej Romaniuk, (2020). Flexible resampling for fuzzy data. International Journal of Applied Mathematics and Computer Science, 30 (2), 281-297.

[9] Reza Ghanbari, Khatere Ghorbani-Moghadam, Nezam Mahdavi-Amiri, (2018). A Direct Method to Compare Bipolar LR-Fuzzy Numbers. Advances in Fuzzy Systems, 1-8, 2018

[10] Rayappan Princy, Krishnaswamy Mohana, (2019). Spherical Bipolar Fuzzy Sets and Its Application in Multi Criteria Decision Making Problem. Journal of New Theory, 32, 58-70.

[11] Reza Ghanbari, Khatere Ghorbani-Moghadam, Nezam Mahdavi-Amiri, (2019). Duality in Bipolar Fuzzy Number Linear Programming Problem. Fuzzy Information and Engineering, 172-185.

[12] Shumaiza, Akram, M., Ahmad N. Al-Kenani, Alcantud, J.C.R. (2019). Group Decision Making Based on the VIKOR Method with Trapezoidal Bipolar Fuzzy Information, Symmetry, 11(10), 1313.

[13] Shumaiza, Akram, M. and Ahmad N. Al-Kenani, (2019). Multiple-Attribute Decision Making ELECTRE II Method under Bipolar Fuzzy Model, Algorithms, 12(11), 226. 